\title{
Correction to: A comprehensive flash flood defense system in China: overview, achievements, and outlook
}

\author{
Liang Guo ${ }^{1,2} \cdot$ Bingshun $\mathrm{He}^{1,2} \cdot$ Meihong $\mathrm{Ma}^{3,4}$. Qingrui Chang ${ }^{1,2}$. Qing $\mathrm{Li}^{1,2}$. \\ Ke Zhang ${ }^{5}$. Yang Hong ${ }^{4,6}$
}

Published online: 20 September 2019

() Springer Nature B.V. 2019

\section{Correction to: Nat Hazards (2018) 92:727-740 https://doi.org/10.1007/s11069-018-3221-3}

The author group of the article ask readers to note the addition of the following affiliation to Yang Hong (co-author):

School of Civil Engineering and Environmental Science, University of Oklahoma. This correction stands to correct the original article. With this correction, the original article has been corrected.

Publisher's Note Springer Nature remains neutral with regard to jurisdictional claims in published maps and institutional affiliations.

The original article can be found online at https://doi.org/10.1007/s11069-018-3221-3.

Bingshun He

hebss@163.com

$\triangle$ Meihong Ma

mmhk12007@163.com

Liang Guo

guol@iwhr.com

Qingrui Chang

changqingrui@126.com

Ke Zhang

kzhang@hhu.edu.cn

Yang Hong

hongyang@tsinghua.edu.cn

1 Research Center on Flood and Drought Disaster Reduction of the MWR, Beijing 100038, China

2 State Key Laboratory of Simulation and Regulation of Water Cycle in River Basin, China Institute of Water Resources and Hydropower Research, Beijing 100038, China

3 Beijing Normal University, Beijing 100875, China

4 State Key Laboratory of Hydroscience and Engineering, Department of Hydraulic Engineering, Tsinghua University, Beijing 100084, China

5 College of Hydrology and Water Resources, Hohai University, Nanjing 210098, Jiangsu Province, China

6 School of Civil Engineering and Environmental Science, University of Oklahoma, Norman, USA 